ARTICLE

\title{
Atomic view of the histidine environment stabilizing higher-pH conformations of $\mathrm{pH}$-dependent proteins
}

Céline Valéry ${ }^{1,2}{ }^{\star}$, Stéphanie Deville-Foillard ${ }^{2,3,4, \star}$, Christelle Lefebvre ${ }^{5}$, Nuria Taberner ${ }^{2}$, Pierre Legrand ${ }^{6}$, Florian Meneau ${ }^{6}$, Cristelle Meriadec ${ }^{5}$, Camille Delvaux ${ }^{3,4}$, Thomas Bizien ${ }^{5}$, Emmanouil Kasotakis ${ }^{3,4}$, Carmen Lopez-Iglesias ${ }^{7}$, Andrew Gall ${ }^{3,4}$, Stéphane Bressanelli ${ }^{3,4}$, Marie-Hélène Le Du ${ }^{3,4}$, Maïté Paternostre ${ }^{3,4} \&$ Franck Artzner ${ }^{5}$

External stimuli are powerful tools that naturally control protein assemblies and functions. For example, during viral entry and exit changes in $\mathrm{pH}$ are known to trigger large protein conformational changes. However, the molecular features stabilizing the higher $\mathrm{pH}$ structures remain unclear. Here we elucidate the conformational change of a self-assembling peptide that forms either small or large nanotubes dependent on the $\mathrm{pH}$. The sub-angstrom high-pH peptide structure reveals a globular conformation stabilized through a strong histidine-serine $\mathrm{H}$-bond and a tight histidine-aromatic packing. Lowering the $\mathrm{pH}$ induces histidine protonation, disrupts these interactions and triggers a large change to an extended $\beta$-sheet-based conformation. Re-visiting available structures of proteins with $\mathrm{pH}$-dependent conformations reveals both histidine-containing aromatic pockets and histidine-serine proximity as key motifs in higher $\mathrm{pH}$ structures. The mechanism discovered in this study may thus be generally used by $\mathrm{pH}$-dependent proteins and opens new prospects in the field of nanomaterials.

\footnotetext{
${ }^{1}$ Biomolecular Interaction Centre, School of Biological Sciences, University of Canterbury, 8140 Christchurch, New zealand. ${ }^{2}$ Ipsen, 5 Avenue du Canada, 91940 Les Ulis, France. ${ }^{3}$ CEA, Institute of Biology and Technologies of Saclay, 91191 CEA-Saclay, France. ${ }^{4}$ Institute for Integrative Biology of the Cell (I2BC), CEA, CNRS, Université Paris-Sud, 91191 CEA-Saclay, Gif sur Yvette, France. ${ }^{5}$ CNRS, UMR 6251, Institut de Physique de Rennes, 263 av. Général Leclerc, Université Rennes I, 35042 Rennes Cedex, France. ${ }^{6}$ Synchrotron SOLEIL, 91190 Gif sur Yvette, France. ${ }^{7}$ Cryo-Electron Microscopy Unit. Scientific and Tecnological Centers of the University of Barcelona, E-08028 Barcelona, Spain. ${ }^{\star}$ These authors contributed equally to this work. Correspondence and requests for materials should be addressed to M.P. (email: maite.paternostre@cea.fr) or to F.A. (email: franck.artzner@univ-rennes1.fr).
} 
$\mathrm{P}$ roteins commonly undergo changes in their three-dimensional (3D) structures to perform their functions. Biologically induced conformational changes can involve motions of groups of atoms or even whole domains of proteins. Changes in protein shape can be triggered by charges, such as calcium and phosphate ${ }^{1}$, or protonation in $\mathrm{pH}$-dependent activities $^{2}$. The detailed comprehension of the underlying molecular mechanisms is an important contribution to the general understanding of protein function. For instance, a wide range of viruses take advantage of the low $\mathrm{pH}$ found in host cell endosomes to sense their cellular location and become activated at the right time and place, for example, to mediate viral and host membrane fusion. The viral proteins responsible for this process have been widely reported to commonly undergo large-scale $\mathrm{pH}$-triggered conformational and oligomerization changes during the membrane fusion process. This is the case for varied pathogens such as influenza virus, vesicular stomatitis virus $^{3,4}$, tick-borne encephalitis virus ${ }^{5,6}$ and dengue ${ }^{7,8}$ virus. Histidine protonation is suspected to be involved in the molecular switch that induces the protein $\mathrm{pH}$-dependent motions. However, experimental demonstrations of single histidines as biomolecular switches have been few, indicating that the precise molecular mechanisms of the protein conformational changes are not well understood, particularly the molecular determinants that stabilize the high-pH protein conformation and trigger its destabilization $^{9,10}$.

In addition, the perfect size monodispersity and the responsiveness of these biological self assemblies correspond to the major challenges for future advanced nanomaterials, that is, (i) the predictable control of the size and morphology of the self-assembled architectures by the chemical structure of the building blocks and (ii) the design of versatile systems that can be triggered by external stimuli. The monodispersity of the final organization and the accuracy of the bio-inspired selfassembly mechanisms have recently attained quasi-perfection with various biological building blocks, such as nucleic acids and amino acids. Any two-dimensional (2D) or 3D nano-object can be designed with DNA origamis ${ }^{11-13}$, while DNA-responsive architectures have been obtained, such as a nano-sized box that can be locked or opened in response to a short-single-stranded $\mathrm{DNA}^{14}$. Responsiveness is, however, limited by the DNA interaction and functionalization possibilities. In this respect, RNA or mixed RNA/DNA origami may be more promising thanks to RNA softness and aptamer recognitions ${ }^{15,16}$. The potential of amino acids for the construction of responsive nanomaterials is correspondingly huge and yet to be tapped. Proteins and peptides are made of building blocks and secondary structures exhibiting a huge variety of interactions and responsive functions. Indeed, proteins $s^{17,18}$ and peptides can be rationally modified to control the self-assembly shapes ${ }^{19-26}$ as well as to finely tune the diameter of nanotubes ${ }^{22-24}$.

In this letter, we report on a decapeptide that self-assembles into either $50 \mathrm{~nm}$ diameter nanotubes at high $\mathrm{pH}$ or $10.7 \mathrm{~nm}$ diameter nanotubes at low $\mathrm{pH}$. The peptide conformation switches from a globular one at $\mathrm{pH}>7.5$ to an extended one at $\mathrm{pH}<6.5$. The high-pH crystal structure obtained at $0.85 \AA$ resolution reveals a histidine-serine $\mathrm{H}$-bond and histidinearomatic interactions, whereas the low-pH molecular structure demonstrates these key interactions have disappeared, likely in favour of cation- $\pi$ proximities. Interestingly, reanalysing protein structures with $\mathrm{pH}$-dependent functions reveals that these specific interaction networks are present in viral, bacterial and human proteins. This molecular switch is demonstrated to have a high potential for the bottom-up design of $\mathrm{pH}$-responsive nanomaterials.

\section{Results}

Conformation transition. Triptorelin is a decapeptide with uncharged $\mathrm{N}$ - and C-termini $\mathrm{pE}_{1}-\mathrm{H}_{2}-\mathrm{W}_{3}-\mathrm{S}_{4}-\mathrm{Y}_{5}-(\mathrm{D}) \mathrm{W}_{6}-\mathrm{L}_{7}-\mathrm{R}_{8}-$ $\mathrm{P}_{9}-\mathrm{G}_{10}-\mathrm{NH}_{2}$ that contains three aromatic side chains $\left(\mathrm{Y}_{5}, \mathrm{~W}_{3}\right.$ and $\left.\mathrm{W}_{6}\right)$, and three ionizable residues, $\mathrm{R}_{8}$ and $\mathrm{Y}_{5}(\mathrm{pKa}>10)$ and a histidine, $\mathrm{H}_{2}(\mathrm{pKa}=6.1$ in water; Supplementary Fig. 1). In the following, the influence of $\mathrm{pH}$ is studied in the range $5-8$, that is, with the $\mathrm{R}_{8}$ remaining positively charged for all the conditions here explored. The triptorelin peptide self-assembles in water into either twisted bundles of small nanotubes at low $\mathrm{pH}$ $(\mathrm{pH}<6.5$, Fig. 1a-f) or large nanotubes at high $\mathrm{pH}(\mathrm{pH}>7.5$, Fig. $1 \mathrm{~g}$ and Supplementary Fig. 3). Given that the peptide single histidine $\left(\mathrm{H}_{2}\right)$ is the only residue with a $\mathrm{pK}_{\mathrm{a}}$ in this range of $\mathrm{pH}$, we explored its protonation state. As shown by the corresponding attenuated total reflectance Fourier-transformed infrared spectroscopy (ATR-FTIR) spectra (Fig. $1 \mathrm{j}$ ), $\mathrm{H}_{2}$ is protonated as a positively charged imidazolium group in the low-pH assemblies (infrared absorption at $1,097 \mathrm{~cm}^{-1}$ ), whereas it is deprotonated in the high-pH assemblies, as a neutral and aromatic $\tau$-tautomer ${ }^{27,28}$ mainly (infrared absorption at $1,106 \mathrm{~cm}^{-1}$, deprotonated histidine on the $\mathrm{N}_{1}$ nitrogen). From one structure to the other, the Fourier-transformed Raman spectroscopy (FT-Raman) evidences a major change in the environments of the aromatic residues and especially tryptophan (Fig. 1h, Supplementary Fig. 2a and Supplementary Note 1). The ATR-FTIR amide I spectra further reveal the presence of $\beta$-sheet hydrogen bond networks in the low-pH assemblies, whereas such networks are only weakly detected in the high-pH ones (Fig. $1 \mathrm{i}$ and Supplementary Fig. $2 \mathrm{~b}$ and Supplementary Note 1). All together, these results show that the peptide conformation, the peptide molecular packing and the assembly morphology of the triptorelin self-assemblies depend on the protonation state of the peptide single histidine, $\mathrm{H}_{2}$ (Fig. 1e).

High-pH nanotubes. At high $\mathrm{pH}\left(\mathrm{pH}>7.5, \mathrm{H}_{2}\right.$ mainly as neutral aromatic $\tau$ tautomer), small-angle X-ray scattering (SAXS) reveals nanotubes with a monodisperse diameter $(\Phi)$ of $500 \AA$ and a wall thickness (e) of $26 \AA$ (Fig. 2a and Supplementary Fig. 3). In some cases, the $2 \mathrm{D}$ hexagonal packing of the nanotubes is observed with a lattice parameter $\left(a_{\text {hex }}\right)$ of $590 \AA$. The organization within the nanotube wall is crystalline as shown by an exceptionally wellaligned wide-angle X-ray scattering pattern (mosaicity $\cong 5^{\circ}$ ) (Fig. 2b and Supplementary Fig. 4). This pattern can be interpreted in terms of a $2 \mathrm{D}$-curved crystal. The position of diffuse scattering maxima can be indexed by a $2 \mathrm{D}$ monoclinic lattice $a=13.7 \AA, b=13.1 \AA, \gamma=88.5^{\circ}$. The line shapes of the diffuse scattering can be simulated by Bessel functions $J_{n}$ with $n=88 \times h+79 \times k$ (Supplementary Fig. 4 and Supplementary Note 2) corresponding to the Fourier transform of a 2D lattice supported by a thin nanotube ${ }^{25}$.

High-pH single crystal. To obtain insights into the nanotube structure at atomic level, the peptide was crystallized as used for amyloid structure determination ${ }^{29}$. The crystallization strategy was inspired by our previous investigations on the lanreotide nanotubes. We reported that specific steric hindrance modifications within the nanotube walls induced predictable variations of the lanreotide nanotube diameter ${ }^{22-24}$. When the lanreotide nanotubes reach a maximal diameter, the system tends to crystallize as flat lamellae packing, that is, with flat walls. Small triptorelin crystals were formed in the presence of phosphate anions that are larger than acetate. By optimizing the crystallization conditions large single crystals diffracting up to $0.85 \AA$ resolution were obtained (Fig. 2e). The $2 \mathrm{D}$-unit cell extracted from the large nanotube X-ray diffraction pattern 
a

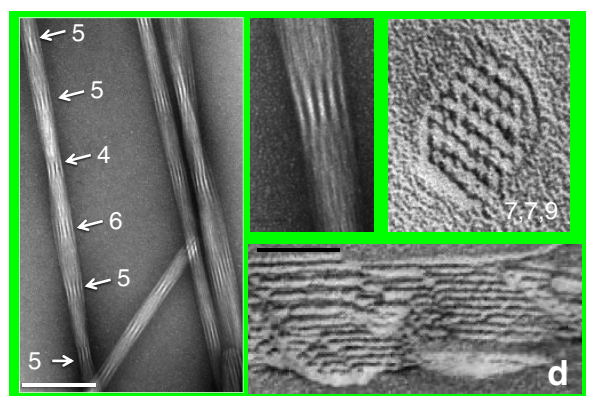

e

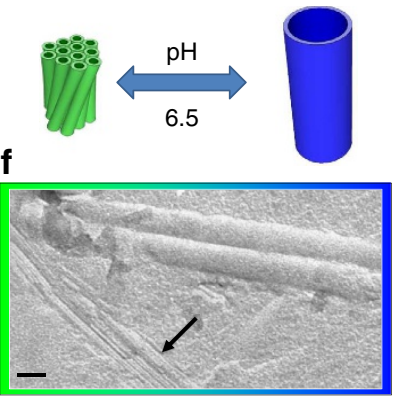

i

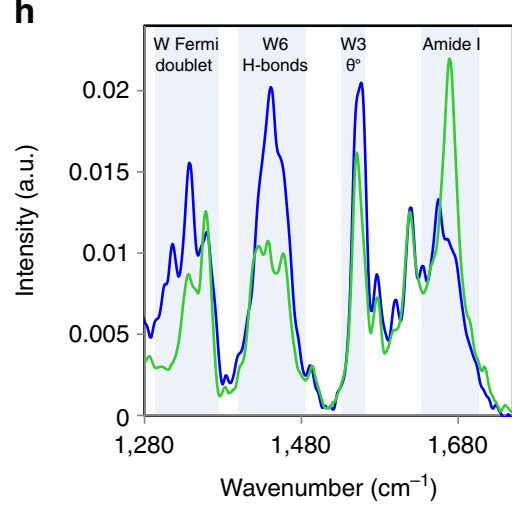

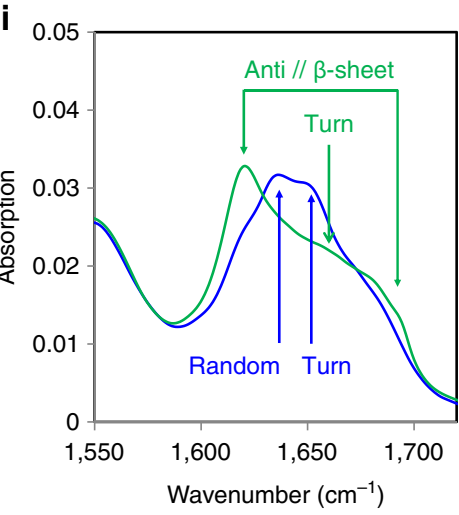

g

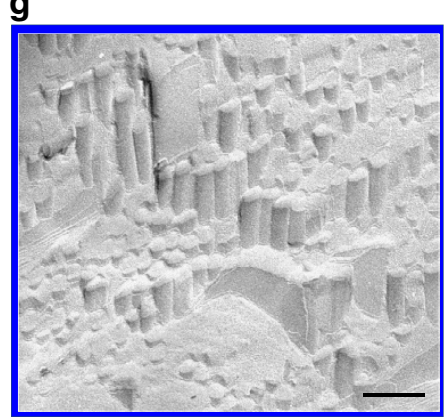

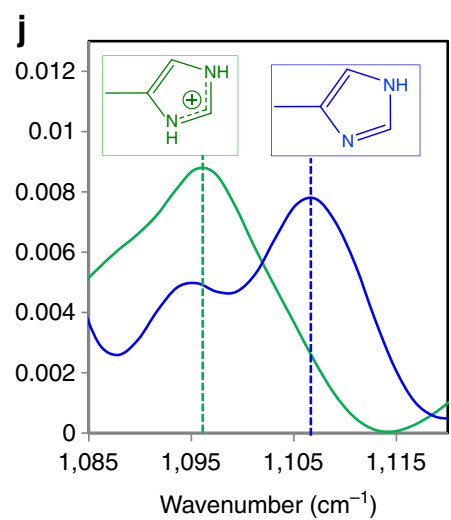

Figure 1 | pH-dependent peptide self-assemblies. (a-d) Electron micrographs of triptorelin self-assemblies formed at low pH (pure water, $\mathrm{pH}$ 6), $(\mathbf{a}, \mathbf{b})$ negative staining and $(\mathbf{c}, \mathbf{d})$ freeze fracture. (a) Bundles of small nanotubes. The white arrows underline on one example the moire patterns on a nanotube bundle that reflect the regular twisting of the nanotubes within the bundles. Near the arrows are written the number of aligned nanotubes that are visible, that is, 5, 5, 4, 6, 5 in this case. (b) Zoom on the moire pattern showing five distinct aligned nanotubes. (c) Zoom on perpendicular fracture of a bundle containing 38 nanotubes. In the direction of the hexagonal packing 7, 7 and 9 alignments of nanotubes are visible. (d) Longitudinal fracture of a nanotube bundle. (e) Scheme underlying the differences of size and packing between a bundle of 12 small (low pH) and 1 large nanotube (high pH). (f) Electron micrographs of freeze-fracture replicas showing the coexistence between bundles of small (black arrow) and large nanotubes. See also Supplementary Fig. 10. (g) Electron micrographs of a freeze fracture replica of triptorelin large nanotubes formed at high $\mathrm{pH}(\mathrm{pH}$ 8.5). (h) Comparison of the FT-Raman spectra of small ( $\mathrm{pH} 6.5$, green) and of large nanotubes ( $\mathrm{pH} \mathrm{8.5,} \mathrm{blue).} \mathrm{The} \mathrm{spectra} \mathrm{are} \mathrm{the} \mathrm{fingerprints} \mathrm{of} \mathrm{the} \mathrm{peptide} \mathrm{environment} \mathrm{and}$ interactions within the assemblies, especially for the environment of the W (Supplementary Fig. 2 and Supplementary Note 1). (i) ATR-FTIR Amide I spectra of small ( $\mathrm{pH} 5.5$, green) and large nanotubes ( $\mathrm{pH} 8.5$, blue). (j) ATR-FTIR spectra of small ( $\mathrm{pH} 5.5$, green) and large nanotubes ( $\mathrm{pH} 8.5$, blue). The absorption bands at 1,097 and $1,106 \mathrm{~cm}^{-1}$ are characteristic to $\left(\mathrm{HisH}_{2}\right)+$ (green) and $\left(\mathrm{His}_{3}\right) \mathrm{H} \tau$ tautomer (blue), that is, protonation of $\mathrm{N}_{3}$, respectively. (electron micrograph scales: $50 \mathrm{~nm}$ ).

$\left(a=13.35 \AA, b=12.93 \AA\right.$ and $\left.\gamma=88.5^{\circ}\right)$ is fully compatible with the layer of the large crystals $(b / 2=13.75 \AA, c=13.1 \AA$ and $\beta=90^{\circ}$ ) (Supplementary Figs 5 and 6, Supplementary Note 3, Supplementary Table 1). Moreover, the micro-Raman spectrum shows that the two $\mathrm{W}$ present in the peptide sequence are in two distinct environments and that there is no $\beta$-sheet network within the three types of assemblies, that is, large nanotubes, small and large crystals (Supplementary Note 4). This information allows us the use of the high-resolution atomic structure obtained by X-ray crystallography to decipher the structure of the wall of the large nanotube. Hence, the 25 - $\AA$-thick bilayer observed in the crystal structure (fully compatible with the $26 \AA$ determined by SAXS) is essentially maintained by aromatic stacking, as shown by the side view of the bilayer (Fig. 2c) and in agreement with the low $\beta$-sheet content observed by ATR-FTIR (Fig. 1j). The histidine tautomer in the crystal and in the nanotubes is the $\tau$ tautomer. Remarkably, the histidine residue is part of a hydrophobic aromatic cluster (Fig. 2f and Supplementary Fig. 6) and its orientation is locked by two strong hydrogen bonds with the $\mathrm{S}_{4} \mathrm{OH}$ group and the backbone amine at distances (nitrogen to proton) of 2.1 and $2.21 \AA$, respectively (Fig. 2e). Finally, in agreement with both ATR-FTIR and Raman data, almost no direct intra- or inter-molecular hydrogen bonds stabilize the peptide conformation or the peptide packing except within the type $\mathrm{II}^{\prime} \beta$-turn induced by the (D) $\mathrm{W}_{6}$.

Low-pH nanotubes. At low pH, the triptorelin assemblies consist of bundles of regularly twisted small nanotubes (diameter $\sim 10 \mathrm{~nm}$ ) (Fig. 1a-d, white arrows on Fig. 1a and zoom on a moiré pattern Fig. $1 \mathrm{~b}$ and Supplementary Fig. 7 and Supplementary Note 5). Despite the lack of a crystal form at low $\mathrm{pH}$, we could infer a reliable low $\mathrm{pH}$, small nanotube molecular model. To that end, we combined structural information from X-ray diffraction patterns (Fig. 3), from ATR-FTIR and Raman spectra (Fig. 1) and from electron microscopy.

The moire patterns within the bundles indicate a well-defined inter-distance between the nanotubes (Fig. 1a,b). The aligned X-ray fibre pattern (Fig. 3a), with a mosaicity corresponding to the bundle twist, exhibits three well-defined scattering planes, an equatorial one and two layers corresponding to $4.85 \AA$ (ref. 31 ) (Fig. 3a). The equatorial diffuse scattering is fitted in terms of poly-disperse bundles of nanotubes with a hexagonal packing of $107 \AA$ (Fig. $3 \mathrm{~b}$ for the model, Supplementary Fig. 8 and Supplementary Note 6). The SAXS peak intensities are in agreement with a wall formed by two shells (Fig. 3b). The 

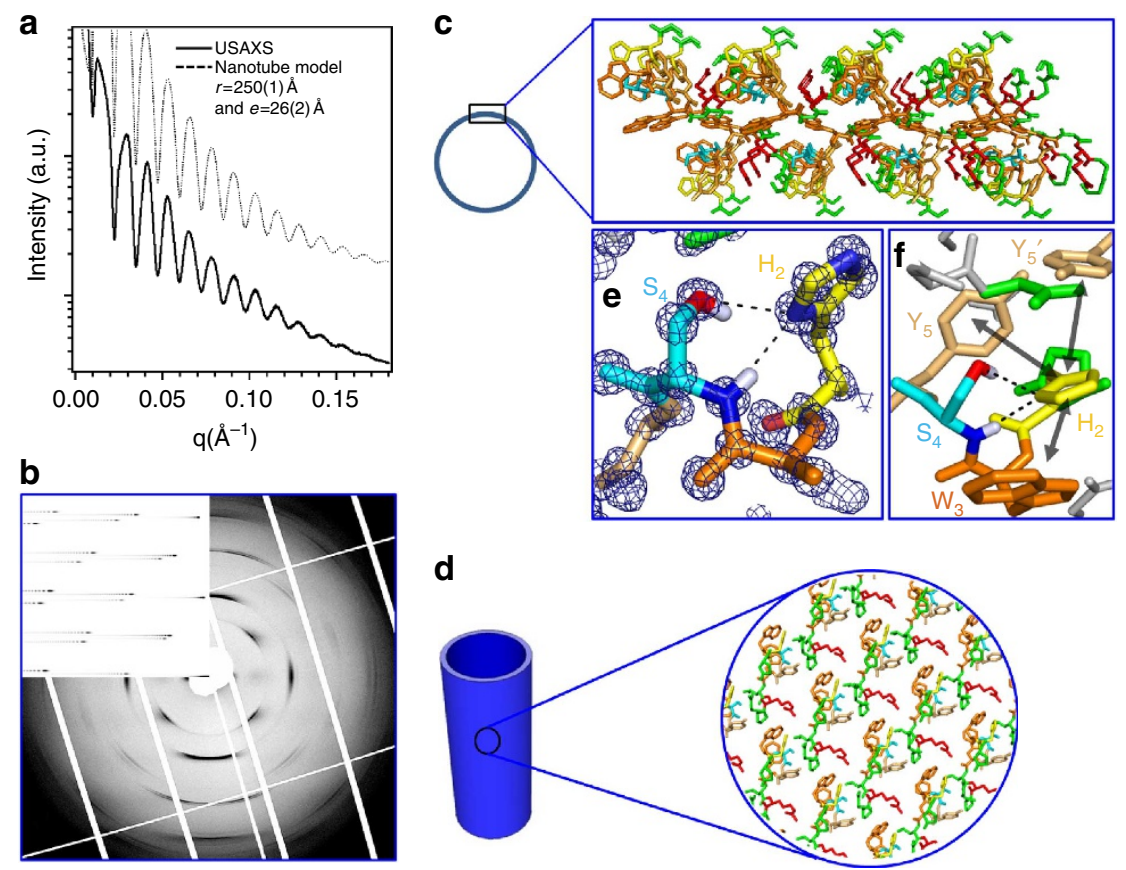

Figure 2 | Atomic structure of the high-pH nanotubes. (a,b) Ultra small angle X-Ray scattering and aligned fibre diffraction pattern of the large nanotubes. $(\mathbf{c}, \mathbf{d})$ Atomic resolution of the peptide organization in the large nanotube wall: side (c) and top (d) views. Colour code: orange (W), light orange ( $\mathrm{Y}$ ), yellow $(H)$, cyan $(S)$ and red $(R)$. The two layers of peptides that form the wall thickness are interacting via an extended aromatic stacking essentially formed by the W side chains. (e) Superposition of the electron density map and structure of the crystal zoomed on the $\mathrm{H}_{2}-\mathrm{S}_{4} \mathrm{H}$-bonds (2.1 $\AA$ between the $\mathrm{N}_{1}$ of the histidine and the proton of the serine $\mathrm{OH}$ group and $2.2 \AA$ between $\mathrm{N}_{1}$ of the histidine and the proton of the amine of the serine backbone). (f) Interaction network around the $\mathrm{H}$ residue (yellow) within the peptide packing: $\mathrm{H}$-bonds between $\mathrm{H}_{2}$ and $\mathrm{S}_{4}$ (black dashed lines) and aromatic cluster around $\mathrm{H}_{2}\left(\mathrm{H}_{2}\right.$-aromatic distance $\left.<4 \AA\right)$ formed by $W_{3}$ and two $Y_{5}$ : intra $\left(Y_{5}\right)$ and inter $\left(Y^{\prime}{ }_{5}\right)$ (black arrows).

nanotube wall full thickness is $26 \AA$ with the outer shell $(10 \AA)$ exhibiting a higher electron density than the inner layer. This indicates that there are more aromatic residues located on the outer surface of the nanotube and that they are involved in the close contact between neighbours. The ATR-FTIR spectra show a turn in the peptide structure (Fig. 1i). Consistently with NMR structure determination of Gonadorelin analogues ${ }^{32}$, this turn is likely localized at the level of the (D) $\mathrm{W}_{6}$. ATR-FTIR spectra further show an extended antiparallel $\beta$-sheet network. The axial position of the $4.85 \AA$ peaks of the X-ray diffraction patterns demonstrates that the $\beta$-sheet $\mathrm{H}$-bond network lies along the nanotube axis and forms protofilaments (Fig. 3a). The additional equatorial diffuse scattering at 8-12 $\AA$ thus corresponds to the interdistance between these protofilaments. The large number of Bragg peaks in the first layer indicates a very high $3 \mathrm{D}$ crystalline sixfold order between nanotubes. This order is only possible with nanotubes that exhibit a six-fold axis, for example, 6n protofilaments per nanotube. Indeed, 30 protofilaments per nanotube yield the correct diameter. The repeat distance along the nanotube axis is the size of the $\beta$-hairpin $9.7 \AA$, for example, $2 \times 4.85 \AA$ (Fig. $3 \mathrm{c}$ for the structure of the protofilament). The systematic absence of three layers $l=2 n+1$ indicates a centred packing, which corresponds to a $4.85 \AA$ translational shift between each protofilament.

From all this information we built the nanotube molecular model and extract the residue close contacts (Fig. 3c,d, Supplementary Fig. 9 and Supplementary Note 6). In this model, the (D) $\mathrm{W}_{6}$ points out of the nanotube. The hexagonal packing of the nanotubes is therefore stabilized by inter-nanotube (D) $\mathrm{W}_{6} \pi$ stacking as confirmed by Raman spectroscopy. Remarkably, at acid $\mathrm{pH}$, the protonated histidine $\mathrm{H}_{2}$ residues are on the inner surface close to $\mathrm{W}_{3}$ and in contact with water.
A key motif of $\mathbf{p H}-d e p e n d e n t$ proteins. The knowledge of both structures that provides the interaction networks stabilizing the high-and low-pH architectures also allows proposing a mechanism of the $\mathrm{pH}$ induced diameter change. At high $\mathrm{pH}$, the monocationic peptide with a single-positive charge on $\mathrm{R}_{8}$ is coiled at the level of the five contiguous residues $\mathrm{H}_{2}-\mathrm{W}_{3}-\mathrm{S}_{4}-\mathrm{Y}_{5}-(\mathrm{D}) \mathrm{W}_{6}$ with five intramolecular interactions among them (Fig. 4, right). At low $\mathrm{pH}$, the repulsive electrostatic forces between the cationic residues, $\left(\mathrm{R}_{8}\right)^{+}$and $\left(\mathrm{H}_{2}\right)^{+}$induce an extended conformation of the $\left(\mathrm{H}_{2}\right)^{+}-\mathrm{W}_{3}-\mathrm{S}_{4}-\mathrm{Y}_{5}-\mathrm{W}_{6}$ sequence of the peptide separating as much as possible the two positive charges on the outer and inner faces of the nanotube wall (Fig. 4, left).

At high $\mathrm{pH}, \mathrm{H}_{2}$ is involved in an intramolecular interaction network, that is, two strong $\mathrm{H}$-bond $\mathrm{H}_{2}-\mathrm{S}_{4}$ and $\pi-\pi$ interactions between $\mathrm{H}_{2}$ and $\mathrm{Y}_{5}$ (intra- and inter-molecularly) and between $\mathrm{H}_{2}$ and $\mathrm{W}_{3}$ (Fig. 2e and 4 right). By decreasing the $\mathrm{pH}$, the histidine group protonates $\left(\mathrm{H}_{2}\right)^{+}$and both $\mathrm{H}$-bond acceptor and aromatic interactions are lost. The coulomb repulsion with $\left(\mathrm{R}_{8}\right)^{+}$ favours the extension of the five contiguous residues $\mathrm{H}_{2}-\mathrm{W}_{3}-\mathrm{S}_{4}-$ $\mathrm{Y}_{5}-(\mathrm{D}) \mathrm{W}_{6}$ to seclude $\left(\mathrm{H}_{2}\right)^{+}$from $\left(\mathrm{R}_{8}\right)^{+}$inducing a $\beta$-hairpin conformation of the peptide. The turn localized around the (D) $\mathrm{W}_{6}$ is maintained and the two cations, $\left(\mathrm{R}_{8}\right)^{+}$and $\left(\mathrm{H}_{2}\right)^{+}$, get close to $\mathrm{W}_{6}$ and $\mathrm{W}_{3}$, respectively, forming possible cation- $\pi$ stabilizing interactions (Fig. 4, left). This large conformational change during the $\mathrm{pH}$ jump induces a major packing modification. The well-packed high-pH bilayer essentially maintained by aromatic residues stacking with almost no intramolecular H-bonding (Fig. 2) turns into a typical amyloid like cross-beta organization at low $\mathrm{pH}$ (Fig. 3). At high $\mathrm{pH}$, the inner and outer leaflets are quasi-similar so that the wall bending is limited and generates large diameter nanotubes. In contrast, at low $\mathrm{pH}$ the high curvature is due to the large steric hindrance mismatch 

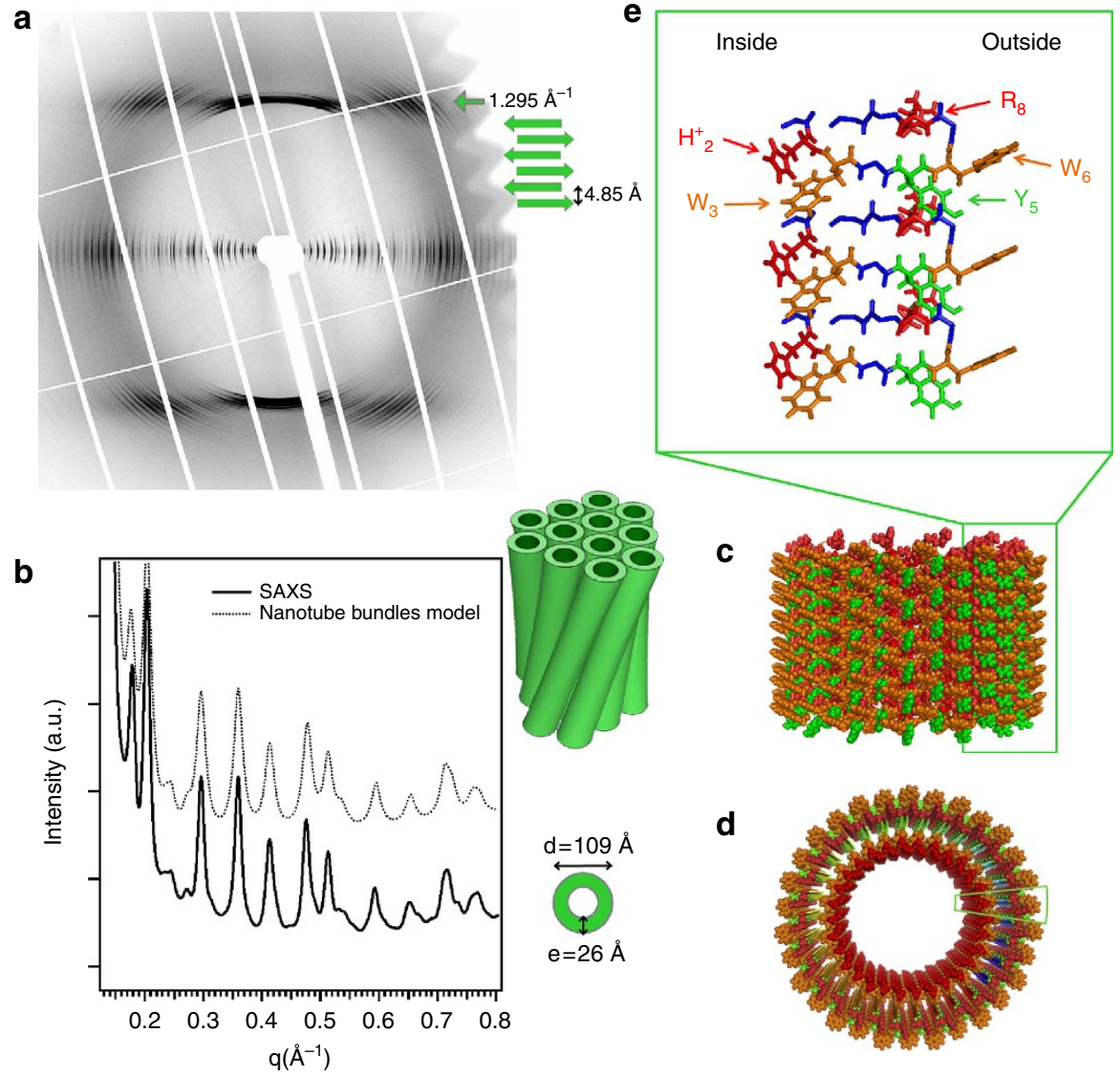

Figure 3 | Molecular structure of the low-pH nanotubes. (a,b) Aligned fibre diffraction (a) and SAXS (b) patterns of triptorelin bundles of small nanotubes. (c) Molecular structure of the protofilaments forming the nanotube walls. Colour code: $W$ (orange), $\mathrm{Y}\left(\right.$ green), protonated histidine $\left(\mathrm{H}_{2}\right)+$ and $\left(R_{8}\right)+($ red). (c,d) Side (c) and top (d) view of the small nanotubes built from 30 protofilaments. The two green boxes underline the position of one protofilament that is detailed in (e).

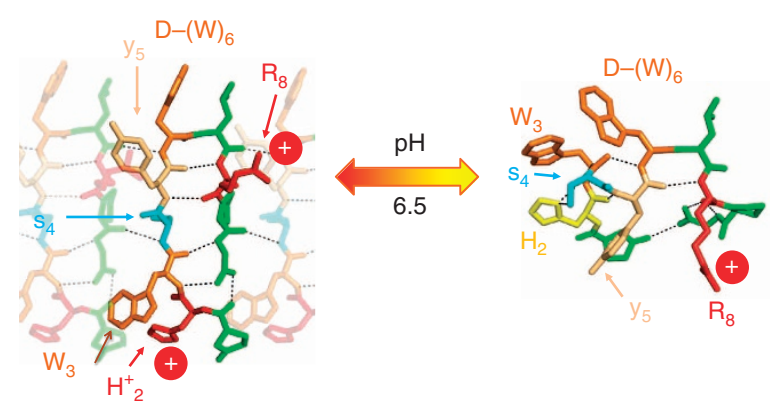

Figure 4 | Peptide conformations. Peptide conformation at low (left) and high (right) $\mathrm{pH}$ : colour codes: W (orange), Y (light orange), S (cyan), R (red), protonated cationic $\mathrm{H}$ (red) and deprotonated neutral and aromatic $\mathrm{H}$ (yellow) (Supplementary Movie 1).

between the two parts of the peptide exposed on the inner and outer faces.

Consequently we here show that the short peptide sequence $\left(\mathrm{H}_{2}-\mathrm{W}_{3}-\mathrm{S}_{4}-\mathrm{Y}_{5}-\mathrm{W}_{6}-\mathrm{L}_{7}-\mathrm{R}_{8}\right)$ folds either as an extended or as a globular conformation depending on $\mathrm{pH}$. The stabilizing forces of the globular folding at high $\mathrm{pH}$ come from both tight $\mathrm{H}$-bonding $\left(\mathrm{H}_{2}-\mathrm{S}_{4}\right)$ and $\pi-\pi$ interactions while the repulsive coulomb interaction between the two cations acts as an extender at low $\mathrm{pH}$. These enforce distinct mono- and dicationic peptide conformations that are both hydrophobic enough to self-organize into curved layers, providing two distinct packings into nanotubes exhibiting consequently two distinct diameters (10.7 vs $50 \mathrm{~nm})$, reversibly switchable by $\mathrm{pH}$ (Supplementary Fig. 10).

\section{Discussion}

The high efficiency of this $\mathrm{pH}$-induced mechanism of peptide conformational switch suggests biological relevance. We therefore asked whether it might regulate proteins whose function requires large conformational changes precisely triggered by a $\mathrm{pH}$ shift. One extensively studied example of such proteins is the diverse families of membrane fusion proteins that enveloped viruses use to enter cells ${ }^{33}$. Many such viral fusion proteins are triggered by the acidification in endosomes after virus internalization. Protonation of histidines has been scrutinized as the triggering mechanism but definite identification of specific $\mathrm{pH}$ sensors has been mostly unsuccessful so far $^{9}, 10$. Strikingly, reanalysis of atomic structures from the PDB and the literature shows that very divergent or even unrelated viral fusion proteins seem to have converged towards combinations of histidine-aromatic clusters and histidine-serine pairs in the key regions that dissociate and/or change structure on lowering of $\mathrm{pH}$ (Table 1, Supplementary Fig. 11 and Supplementary Note 7). The evidence for this aromatic-histine-serine switch could be extended to other types of conformationally $\mathrm{pH}$-sensitive proteins. Indeed, the structures of the $\mathrm{pH}$-dependent bacterial membrane channel $\mathrm{OmpG}^{34}$ and of the soluble protein $\mathrm{M}$-ficolin ${ }^{35}$ also support this motif as involved in the mechanisms of $\mathrm{pH}$-dependent conformational change (Table 1, Supplementary Fig. 11 and Supplementary Note 7). This non-exhaustive protein structure analysis shows that aromatic pockets and histidine-serine $\mathrm{H}$-bonds may be used by a wide 
Table 1 | Examples of pH-sensitive proteins showing histidine-serine H-bonds and histidine-aromatic pockets in the high-pH structures that are absent in the low-pH ones.

\begin{tabular}{|c|c|c|c|c|c|c|}
\hline $\begin{array}{l}\text { Prot. name PDB } \\
\text { ID basic(acid) }\end{array}$ & Protein family & $\begin{array}{l}\text { Resolution } \\
\text { basic(acid) }\end{array}$ & $\begin{array}{l}\text { H-bond } \\
\text { residues }\end{array}$ & $\begin{array}{l}\text { H-bond distances } \\
\text { basic(acid) }\end{array}$ & $\begin{array}{l}\pi / \pi \text { interactions } \\
\text { residues }\end{array}$ & $\begin{array}{l}\pi / \pi \text { interactions } \\
\text { distances basic(acid) }\end{array}$ \\
\hline 3J27@(1URZ) & $\begin{array}{l}\text { Membrane fusion protein } \\
\text { Flavivirus Class II }\end{array}$ & $3.5 \AA(2.7 \AA)$ & $\mathrm{H}_{317^{\star}}{ }^{\star} \mathrm{S}_{396}{ }^{\star}$ & $3.3 \AA(14.0 \AA)$ & $\begin{array}{l}\mathrm{H}_{261}-\mathrm{W}_{19}^{\dagger} \\
\mathrm{H}_{7}^{\dagger}-\mathrm{W}_{212}\end{array}$ & $\begin{array}{l}3.6 \AA^{\ddagger}\left(N / A^{\dagger}\right) \\
2.8 \AA^{\ddagger}\left(N / A^{\dagger}\right)\end{array}$ \\
\hline $3 N 43\left(N / A^{\dagger}\right)$ & $\begin{array}{l}\text { Membrane fusion protein } \\
\text { Alphavirus Class II }\end{array}$ & $2.6 \AA\left(N / A^{\dagger}\right)$ & $\mathrm{H}_{170^{\star}, \dagger}-\mathrm{S}_{57}{ }^{\star}$ & $2.9 \AA\left(N / A^{\dagger}\right)$ & $\mathrm{H}_{73}^{\dagger}-\mathrm{W}_{89}$ & $3.6 \AA^{\ddagger}\left(N / A^{\dagger}\right)$ \\
\hline $2 J 6 J(2 C M Z)$ & $\begin{array}{l}\text { Membrane fusion protein } \\
\text { Rhabdovirus Class III }\end{array}$ & $3 \AA(2.4 \AA)$ & $\mathrm{H}_{407}{ }^{\star}-\mathrm{S}_{84}{ }^{\star}$ & $3.4 \AA(32.2 \AA)$ & $\mathrm{H}_{33}-\mathrm{F}_{189}$ & $3.9 \AA \ddagger(11.2 \AA)$ \\
\hline OmpG 2IWW(2IWV) & Bacterial membrane protein & $2.3 \AA(2.7 \AA)$ & $\mathrm{H}_{231}-\mathrm{S}_{218}$ & $2.9 \AA(9.9 \AA)$ & $\mathrm{H}_{261}-\mathrm{F}_{233}$ & $4.5 \AA(7,9 \AA)$ \\
\hline M-Ficolin 2JHM(2JHH) & Human soluble protein & $1.52 \AA(1.7 \AA)$ & $\begin{array}{l}\mathrm{H}_{268}-\mathrm{S}_{270} \\
\mathrm{H}_{222}-\mathrm{S}_{257}\end{array}$ & $\begin{array}{l}3.5 \AA(8.7 \AA) \\
3.6 \AA(12.7 \AA)\end{array}$ & $\begin{array}{l}\mathrm{H}_{222}-\mathrm{W}_{249} \\
\mathrm{H}_{255}-\mathrm{Y}_{251} \\
\mathrm{H}_{255}-\mathrm{Y}_{283}\end{array}$ & $\begin{array}{l}3.6 \AA(3.3 \AA) \\
4.0 \AA(10.5 \AA) \\
3.6 \AA(17.5 \AA)\end{array}$ \\
\hline \multicolumn{7}{|c|}{$\begin{array}{l}\text { (Supplementary Fig. } 11 \text { and Supplementary Note } 7 \text { ) } \\
@ \text { Cryo EM structure of the native virion. } \\
\text { 'These residues are conserved within each viral genus despite low overall sequence identity. } \\
\text { †In the prefusion assembly these interacting residues are harboured by a companion protein that dissociates from the fusion protein on exposure to low pH. } \\
\text { In all cases of viral fusion proteins, in the prefusion conformation there is at least one histidine-containing aromatic cluster that is conserved (although individual residues may not be) and that is } \\
\text { disrupted in the postfusion conformation. The aromatic residue closest to the histidine is given. }\end{array}$} \\
\hline
\end{tabular}

variety of proteins with a biological function that requires a $\mathrm{pH}$ induced conformational switch.

Beyond the biological relevance, this mechanism opens significant downstream perspective in the field of nanomaterials. Indeed, there are very few self-assemblies having well-defined organizations with peptides ${ }^{20,25,30,36}$, proteins ${ }^{37}$ or DNA $^{11}$ and even less that can be continuously tuned $22,24,38$. One major challenge is the design of systems that can be triggered by external stimuli such as the $\mathrm{pH}^{21,39,40}$. Our example of $\mathrm{pH}$-responsive nanotube diameter is based on a very short peptide sequence of 10 amino acids. The chemical variety of amino acids opens easier and richer functionalization perspectives than other advanced bio-inspired self-assemblies ${ }^{11}$.

In conclusion, we decipher the atomic details of $\mathrm{pH}$-tuned alternate conformations that result in large self-assembly differences. The basic conformation is stabilized by a highly specific histidine-serine $\mathrm{H}$-bond and by histidine-aromatic stacking. The acid conformation is imposed by the repulsive coulomb interactions between both cationic protonated histidine and arginine. This molecular switch is general, as we have found such switches in a large range of proteins including human, viral and bacterial proteins either soluble or insoluble. The conformational molecular switch described here may also be involved in protein folding ${ }^{41}$. Finally, this mechanism opens prospects in the field of nanomaterials where systems that can be reversibly triggered by external stimuli are highly desirable.

\section{Methods}

Triptorelin peptide. (pE1-H2-W3-S4-Y5-(D)W6-L7-R8-P9-G10- $\mathrm{NH}_{2}$ ) or (pGlu-His-Trp-Ser-Tyr-D-Trp-Leu-Arg-Pro-Gly- $\mathrm{NH}_{2}$ ) or 5-oxo-D-prolyl-Lhistidyl-L-tryptophyl-L-seryl-L-tyrosyl-3-(1H-indol-2-yl)-L-alanylleucyl-L-arginyl-L-prolylglycinamide was provided by IPSEN as lyophilized powder and acetate salt (purity $>99 \%$; water content $20 \%$ and acetate/peptide (MW/MW) ratio is 1.8 ).

Raman microscopy (micro-Raman). Room-temperature Raman spectra were recorded with a Jobin Yvon U1000 spectrometer equipped with a Symphony CCD camera coupled to a BX41 Olympus microscope. Excitation at $514.5 \mathrm{~nm}$ was provided by Coherent Argon (Innova 100) laser. No evolution of the resonance Raman signal was observed during data acquisition.

FT-Raman. Spectra were recorded at $4 \mathrm{~cm}^{-1}$ resolution using a Bruker IFS66 interferometer coupled to a Bruker FRA106 Raman module equipped with a continuous Nd: Yag laser providing excitation at $1,064 \mathrm{~nm}$. All spectra were recorded at room temperature with backscattering geometry from samples in hermetically closed glass capillaries. The spectra resulted from 500 to $5,000 \mathrm{~cm}^{-1}$.

ATR-FTIR. ATR-FTIR spectra were recorded at a $4 \mathrm{~cm}^{-1}$ resolution with a Bruker IFS 66 spectrophotometer equipped with a $45^{\circ} \mathrm{N}$ ZnSe ATR attachment. The spectra obtained resulted from the average of 30 scans and were corrected for the linear dependence on the wavelength of the absorption measured by ATR. The water signal was removed by subtraction of pure water spectrum.

Optical microscopy. Optical microscopy observations were performed using an Axiovert $100 \mathrm{~A}$ Olympus inverted microscope, at magnifications up to 20 (A-plan Olympus objectives), on samples conditioned in thin layers between glass coverslips. The polarizer and analyser were in a fixed crossed position. A retardation plate was inserted at $45^{\circ}$ to the polarizers. Digital images were taken with an Olympus Highlight 3000 camera connected to a computer.

Sample preparation for electron microscopy. Negatively stained samples: drops of triptoreline acetate solutions were deposited on formvar-coated electron microscopy grids. After 1 min contact, the excess of water was removed and a drop of $1 \%$ uranyl acetate was deposited for $1 \mathrm{~min}$ on the top of the sample. The excess of uranyl acetate was removed.

Preparation of the sample for metal replica processing: different techniques for sample fracturing were used: (i) drops of triptoreline acetate solutions were sandwiched between two copper platelets using a 400-mesh gold grid as a spacer. The samples were then frozen at $-190^{\circ} \mathrm{C}$ by rapid immersion in liquid propane and stored at $-196^{\circ} \mathrm{C}$ in liquid nitrogen until subsequent use. Frozen samples were fractured by opening the two copper platelets at $-150^{\circ} \mathrm{C}$ and $2 \times 10^{-8} \mathrm{mbar}$ in a Baltec BAF 060 freeze-fracture apparatus (BAL-TEC, Liechtenstein), (ii) drops of triptoreline were directly frozen at $-190^{\circ} \mathrm{C}$ by rapid immersion in liquid propane and stored at $-196^{\circ} \mathrm{C}$ in liquid nitrogen until subsequent use. Frozen samples were further fractured by microtomy inside the Baltec BAF 060 freezefracture apparatus (BAL-TEC, Liechtenstein). No cryo-protectant was necessary for these preparations.

Freeze-etching was carried out by switching the sample temperature from -150 to $-100^{\circ} \mathrm{C}$ and maintaining the pressure at $10^{-7} \mathrm{mbar}$ for $2 \mathrm{~min}$ to sublimate the surface layer of ice.

Metal replicas of the exposed surfaces (from the different preparations described) were obtained by evaporating $2 \mathrm{~nm}$ of platinum with an electron gun at an angle of $45^{\circ}$ and $20 \mathrm{~nm}$ of carbon with an electron gun at an angle of $90^{\circ}$. After being floated in distilled water, the replicas were picked up on formvar-coated electron microscopy grids.

Electron microscopy. Either stained samples or freeze-fracture replicas, which were previously deposited on 200-mesh formvar-coated copper grids, were examined with either a JEOL 1,010 or a Philips CM12 transmission electron microscope at $80 \mathrm{kV}$.

SAXS, fibre diffraction, X-ray diffraction. SAXS: this was performed on the high brilliance SWING beam line $(12 \mathrm{KeV})$ at the Soleil Synchrotron Facility using 
sample-detector distances of $0.5-3 \mathrm{~m}$ depending on the experiments. The diffraction patterns were therefore recorded for reciprocal spacing $q\left(\AA^{-1}\right)$

from 0.007 to $2.1 \AA^{-1}$, that is, a range of repetitive distances $d=2 \pi / q$ from 990 and $3 \AA$. The X-ray patterns were detected and recorded via a chip charge-coupled device camera detector, AVIEX. The samples were prepared in 1.3 to $1.6 \mathrm{~mm}$ glass capillaries (Glas Technik and Konstruktion, Schönewalde, Germany) and introduced into a homemade capillary holder accommodating 20 capillaries at controlled temperature. All samples exhibited powder diffraction and scattering intensities as a function of the radial wave vector, $q=4 \pi \sin (\theta / \lambda)$, which was determined by circular integration.

Crystal and fibre X-Ray diffraction were performed on PROXIMA1 (Soleil) beamline using a Pilatus $6 \mathrm{M}$ detector. For crystal diffraction the wavelength was fixed at $0.78471 \AA$. For fibre diffraction the wavelength was fixed at $0.98011 \AA$ and the detector to sample distance at $0.3202 \mathrm{~m}$. A homemade sample holder adapted built for PX1 beam line maintained the capillary.

Fibre growth on magnetic field gradient. The bundles of nanotubes obtained from the self-assembly of triptoreline at low $\mathrm{pH}$ were grown in a strong magnetic field gradient on the top of a small $\left(1 \mathrm{~cm}^{3}\right)$ rare-earth magnets (gift from Sakellariou Dimitrious, DSM/IRAMIS, Cea-Saclay). In this condition, very well-aligned fibres were obtained and analysed by wide-angle X-ray scattering on SWING and PROXIMA1 beamlines (Soleil).

Triptoreline crystallization. Lyophilized triptoreline was dissolved in water and subjected to standard crystallization screens, by sitting drop and batch methods. Crystallization drops were examined by optical microscopy over times ranging from a few minutes to a few weeks.

Triptoreline monocrystals were obtained in the presence of phosphate buffer. The first crystallization screening shows that small peptide crystals were obtained in a large range of peptide concentration $(0.5-8 \%(\mathrm{w} / \mathrm{w})$ ) and of $\mathrm{pH}$ buffer (from 6 to 8). To enforce the crystal size growing, we rationalized that we should decrease the crystallization kinetic by working at a $\mathrm{pH}$ close to the Histidine pKa. Indeed, the optimized conditions for crystal growth were found at $\mathrm{pH} 6.2$.

More precisely, the monocrystals of triptorelin were obtained when the reservoir contained $500 \mu \mathrm{l}$ of $300 \mathrm{mM}$ potassium phosphate buffer, $\mathrm{pH} 6.2$ close to the pKa (6.1) determined for the non-assembled peptide (Supplementary Fig. 1b). The drop was a mixture of $10-\mu \mathrm{l}$ reservoir solution and $10 \mu \mathrm{l}$ peptide solution $\left(80 \mathrm{mg} \mathrm{ml}^{-1}\right.$ triptoreline in water) ( $4 \%$ for the final peptide concentration and $150 \mathrm{mM}$ for the phosphate buffer). The reservoir was filled before the drop. The drop was prepared by quick pipette addition of the peptide solution to the reservoir solution. Crystals were grown at $20^{\circ} \mathrm{C}$. Crystals were transferred to a cryoprotectant (25\% glycerol in $300 \mathrm{mM}$ potassium phosphate buffer $\mathrm{pH} 6.2$ ) before cryogenic processing and data collection.

Single-crystal structure determination. Ultra-high resolution data were collected to $0.85 \AA$ on Proximal beamline at Soleil synchrotron. To reach this resolution, the beam wavelength was adjusted to $0.78471 \AA$. The X-Ray diffraction pattern recorded for triptorelin mono crystal is shown in Supplementary Fig. 5b. Data integration and scaling were performed using the XDS package642. The structure of triptorelin was solved by dual-space direct methods with SHELXD43. The model was completed by alternative cycles of manual model building with Coot 44 and refinement with REMAC545. The data statistics of the triptorelin structure determination are indicated on Supplementary Table 1.

\section{References}

1. Clapham, D. E. Calcium signaling. Cell 131, 1047-1058 (2007).

2. Srivastava, J., Barber, D. L. \& Jacobson, M. P. Intracellular $\mathrm{pH}$ sensors: design principles and functional significance. Physiology 22, 30-39 (2007).

3. Roche, S., Bressanelli, S., Rey, F. A. \& Gaudin, Y. Crystal structure of the low-pH form of the vesicular stomatitis virus glycoprotein G. Science 313, 187-191 (2006).

4. Roche, S., Rey, F. A., Gaudin, Y. \& Bressanelli, S. Structure of the prefusion form of the vesicular stomatitis virus glycoprotein G. Science 315, 843-848 (2007).

5. Bressanelli, S. et al. Structure of a flavivirus envelope glycoprotein in its lowpH-induced membrane fusion conformation. EMBO J. 23, 728-738 (2004).

6. Rey, F. A., Heinz, F. X., Mandl, C., Kunz, C. \& Harrison, S. C. The envelope glycoprotein from tick-borne encephalitis-virus at 2 A resolution. Nature 375, 291-298 (1995).

7. Modis, Y., Ogata, S., Clements, D. \& Harrison, S. C. A ligand-binding pocket in the dengue virus envelope glycoprotein. Proc. Natl Acad. Sci. USA 100, 6986-6991 (2003).

8. Modis, Y., Ogata, S., Clements, D. \& Harrison, S. C. Structure of the dengue virus envelope protein after membrane fusion. Nature 427, 313-319 (2004).

9. Harrison, J. S. et al. Role of electrostatic repulsion in controlling $\mathrm{pH}$-dependent conformational changes of viral fusion proteins. Structure 21, 1085-1096 (2013).

10. Harrison, S. C. The $\mathrm{pH}$ sensor for flavivirus membrane fusion. J. Cell Biol. 183, 177-179 (2008)

11. Rothemund, P. W. K. Folding DNA to create nanoscale shapes and patterns. Nature 440, 297-302 (2006).
12. Han, D. R. et al. DNA origami with complex curvatures in three-dimensional space. Science 332, 342-346 (2011).

13. Lin, C., Liu, Y. \& Yan, H. Designer DNA nanoarchitectures. Biochemistry 48, 1663-1674 (2009).

14. Andersen, E. S. et al. Self-assembly of a nanoscale DNA box with a controllable lid. Nature 459, 73-76 (2009).

15. Chworos, A. et al. Building programmable jigsaw puzzles with RNA. Science 306, 2068-2072 (2004).

16. Delebecque, C. J., Lindner, A. B., Silver, P. A. \& Aldaye, F. A. Organization of intracellular reactions with rationally designed RNA assemblies. Science 333, 470-474 (2011).

17. Ardejani, M. S., Chok, X. L., Foo, C. J. \& Orner, B. P. Complete shift of ferritin oligomerization toward nanocage assembly via engineered protein-protein interactions. Chem. Commun. 49, 3528-3530 (2013).

18. Lai, Y. T., Cascio, D. \& Yeates, T. O. Structure of a 16-nm cage designed by using protein oligomers. Science 336, 1129-1129 (2012).

19. Ardejani, M. S. \& Orner, B. P. Obey the peptide assembly rules. Science 340, 561-562 (2013).

20. Fletcher, J. M. et al. Self-assembling cages from coiled-coil peptide modules. Science 340, 595-599 (2013).

21. Hartgerink, J. D., Beniash, E. \& Stupp, S. I. Peptide-amphiphile nanofibers: a versatile scaffold for the preparation of self-assembling materials. Proc. Natl Acad. Sci. USA 99, 5133-5138 (2002).

22. Tarabout, C. et al. Control of peptide nanotube diameter by chemical modifications of an aromatic residue involved in a single close contact. Proc. Natl Acad. Sci. USA 108, 7679-7684 (2011).

23. Gobeaux, F. et al. Directing peptide crystallization through curvature control of nantubes. J. Pept. Sci. 20, 508-516 (2014).

24. Gobeaux, F. et al. Structural role of counterions adsorbed on self-assembled peptide nanotubes. J. Am. Chem. Soc. 134, 723-733 (2012).

25. Valery, C. et al. Biomimetic organization: octapeptide self-assembly into nanotubes of viral capsid-like dimension. Proc. Natl Acad. Sci. USA 100, 10258-10262 (2003).

26. Valery, C. et al. Molecular origin of the self-assembly of lanreotide into nanotubes: a mutational approach. Biophys. J. 94, 1782-1795 (2008).

27. Barth, A. The infrared absorption of amino acid side chains. Prog. Biophys. Mol. Biol. 74, 141-173 (2000).

28. Li, S. H. \& Hong, M. Protonation, tautomerization, and rotameric structure of histidine: a comprehensive study by magic-angle-spinning solid-state NMR. J. Am. Chem. Soc. 133, 1534-1544 (2011).

29. Colletier, J.-P. et al. Molecular basis for amyloid-beta polymorphism. Proc. Natl Acad. Sci. USA 108, 16938-16943 (2011).

30. Pouget, E. et al. Elucidation of the self-assembly pathway of lanreotide octapeptide into beta-sheet nanotubes: role of two stable intermediates. J. Am. Chem. Soc. 132, 4230-4241 (2010).

31. Vainshtein, B. K. Diffraction of X-rays by chain molecules (Elsevier, 1966).

32. Laimou, D. K. et al. Structural elucidation of Leuprolide and its analogues in solution: insight into their bioactive conformation. Amino Acids 39, 1147-1160 (2010).

33. Albertini, A., Bressanelli, S., Lepault, J. \& Gaudin, Y. in Membrane Fusion Vol. 68 Current Topics in Membranes 49-80 (Elsevier Academic Press Inc, 2011).

34. Yildiz, O., Vinothkumar, K. R., Goswami, P. \& Kuhlbrandt, W. Structure of the monomeric outer-membrane porin OmpG in the open and closed conformation. EMBO J. 25, 3702-3713 (2006).

35. Garlatti, V. et al. Structural basis for innate immune sensing by M-ficolin and its control by a $\mathrm{pH}$-dependent conformational switch. J. Biol. Chem. 282, 35814-35820 (2007).

36. Gobeaux, F. et al. Experimental observation of double-walled peptide nanotubes and monodispersity modeling of the number of walls. Langmuir. 29, 2739-2745 (2013).

37. King, N. P. et al. Computational design of self-assembling protein nanomaterials with atomic level accuracy. Science 336, 1171-1174 (2012).

38. Oda, R., Huc, I., Schmutz, M., Candau, S. J. \& MacKintosh, F. C. Tuning bilayer twist using chiral counterions. Nature 399, 566-569 (1999).

39. Banerjee, I. A., Yu, L. T. \& Matsui, H. Cu nanocrystal growth on peptide nanotubes by biomineralization: Size control of $\mathrm{Cu}$ nanocrystals by tuning peptide conformation. Proc. Natl Acad. Sci. USA 100, 14678-14682 (2003).

40. Li, B. S. et al. Tuning the chain helicity and organizational morphology of an L-valine-containing polyacetylene by $\mathrm{pH}$ change. Nano Lett. 1, 323-328 (2001). 41. Dobson, C. M. Protein folding and misfolding. Nature 426, 884-890 (2003).

\section{Acknowledgements}

Merce Cardus discovered the self-assembly properties of Triptorelin. We acknowledge Juliet Gerrard, Loic Toupet and Alberto Mezzetti for discussions. Dimitrious Sakellariou is acknowledged for the rare-earth magnet gift and discussions on magnetic field and magnetic field gradient. Jean-claude Ameline is acknowledged for technical support. This work was supported by the 'Région Bretagne', the 'ANR-Biotech SmartPep', the 'French Infrastructure for Integrated Structural Biology' (FRISBI) ANR-10-INSB-05-01 and the French/New Zealand Dumont d’Urville grant $N^{\circ} 27788$ WE. Synchrotron Soleil (France) 
is acknowledged for beam time allocation (proposal 20120497 and BAG 'Structural studies of proteins of medical interest’ $\mathrm{N}^{\circ} 20120651$ ). The mini electron microscopy platform of iBiTec-S, TEM team is acknowledged.

\section{Author contributions}

S.D.-F., C.L., N.T., M.C., C.D. and E.K. performed the experiments; P.L. and F.M. assisted in X-Ray data collection at synchrotron soleil (beamlines Proximal and Swing, respectively); C.M. and T.B. performed the X-Ray experiments in Rennes and at synchrotron Soleil; P.L. and M.-H.L. solved the peptide crystal structure at high pH; M.-H.L. and S.D.-F. did the crystallogenesis; C.L.I. did the freeze fracture for the large nanotubes; S.B. reanalysed the structures of the $\mathrm{pH}$-sensitive proteins; C.V. designed and performed the experiments. F.A. and M.P. designed, performed and interpreted the experiments.

\section{Additional information}

Accession codes: Atomic coordinates and structure factors for the reported crystal structures have been deposited with the Protein Data Bank (http://www.pdb.org/) under accession code $4 \mathrm{D} 5 \mathrm{M}$.
Supplementary Information accompanies this paper at http://www.nature.com/ naturecommunications

Competing financial interests: The authors declare no competing financial interests.

Reprints and permission information is available online at http://npg.nature.com/ reprintsandpermissions/

How to cite this article: Valéry, C. et al. Atomic view of the histidine environment stabilizing higher $\mathrm{pH}$ conformations of $\mathrm{pH}$-dependent proteins. Nat. Commun. 6:7771 doi: 10.1038/ncomms8771 (2015).

(c) (i) This work is licensed under a Creative Commons Attribution 4.0 International License. The images or other third party material in this article are included in the article's Creative Commons license, unless indicated otherwise in the credit line; if the material is not included under the Creative Commons license, users will need to obtain permission from the license holder to reproduce the material. To view a copy of this license, visit http://creativecommons.org/licenses/by/4.0/ 